\title{
UWB signal detection based on wavelet packet and FHN model
}

\author{
Bowen Chen ${ }^{*}$, Lei Jiang, Qun Zhang \\ Information and Navigation College, Air Force Engineering University, \\ Feng Hao Road, Xi'an, Shaanxi \\ Xi'an, 710077, China \\ E-mail: cbw0709@126.com
}

\begin{abstract}
In UWB-IR signal detection, the threshold of signal to noise ratio(SNR) limit the performance of FHN model detection method, from this point, wavelet packet is introduced into FHN model, a novel UWB-IR signal detection method based on wavelet packet and FHN model is proposed, in addition, the disadvantages of traditional single threshold wavelet packet is analyzed, combined with the new piecewise threshold wavelet packet and FHN model to detect UWB-IR signal. Furthermore, the performance of the proposed algorithm is simulated and analyzed. Simulation results shows that the proposed algorithm overcome the SNR threshold of FHN model detection method, the detection performance of FHN model is improved. Therefore, the UWB-IR signal can be detected effectively under strong noise.
\end{abstract}

Keywords: ultra wideband; signal detection; wavelet packet; FHN; stochastic resonance; piecewise threshold

\section{Introduction}

Ultra Wideband-Impulse Radio(UWB-IR) is a kind of communication signal which is modulated by ultra-short duration pulse directly, it can realize high speed data transmission, high precision ranging and strong penetration[1], with the excellent properties mentioned above, ultra wideband technology has great potential in wireless Ad hoc network[2], Wireless LAN[3] and RF stealth[4] and other fields. In addition, the strong antijamming ability and low interception/detection probability(LPI/D) characteristics of ultra wideband communication make it highly concerned by military departments of various countries, some product such as C-band collision avoidance radar for UAV, military
UWB communication radio station in VHF band, Lband altimeter, UWB high speed data link and active positioning device has emerged[5]. However, the low level power spectrum density of UWB-IR signal lead to the wanted signal that submerged in noise is difficult to detect in the long range communication or complex channel environment, which brings a great challenge to the non-cooperative third part to reconnoitre signal. Therefore, the effective detection method of UWB-IR signal is very important in electronic warfare[6].

At present, the detection method of UWB-IR signal mainly focus on the signal correlation characteristic, Hilbert-Huang transform(HHT), time-frequency analysis and stochastic resonance(SR). However, the correlation detection mainly used for the DS-UWB

\footnotetext{
${ }^{*}$ Corresponding author
} 
signal, it is not suitable for the TH-UWB signal[7]; the detection performance HHT is greatly affected by the noise, the reconstructed waveform has a lower fitting degree to the original UWB-IR signal under the strong noise[8]; time frequency analysis is not suitable for the UWB-IR signal detection under low SNR[9]; SR can be used to detect the UWB-IR signal under the strong noise.

The basic idea of SR is amplify the weak wanted component by the noise in the signal[10], it has been widely applied in the field of weak signal detection. Unfortunately, the research of stochastic resonance is mostly focused on the periodic signal at present, however, most signal in practical application is aperiodic signals. To overcome this disadvantage, FHN neuron model stochastic resonance is proposed by Collins et al in 1995[11], it provides a new method for people to study the aperiodic signal detection. With years of development, it is proved that FHN model can be used to detect UWB-IR signal[12], but the detection SNR is limited to $-20 \mathrm{~dB}$.

Wavelet packet is a generalization of wavelet, compared with the wavelet denoising method, wavelet packet denoising consider the useful information in each frequency band at the same time, which will improve the precision of denoising to the medium and high frequency information. Based on this method, the signal can be further removed from the noise in the signal, so it is more targeted to UWB-IR signal. With the further research of wavelet packet, it is found that the selection of threshold criterion is the key to the denoising performance[13]. The traditional threshold selection criterion mostly is the single threshold criterion, however, different threshold criteria have different sensitivity to each frequency band, single threshold criterion can not consider the frequency distribution of signal and noise fully, so the denoising precision need to be further improved.

In view of this, in order to overcome the limitation of SNR that detect UWB-IR signal by using FHN model, a joint detection method based on wavelet packet and FHN model is proposed to realize UWB-IR signal detection under lower SNR. Furthermore, a new piecewise threshold wavelet packet is proposed to overcome the shortcoming of single threshold criterion, that is, take a different threshold criteria to different frequency bands according to the characteristics of different frequency bands. Combined with piecewise threshold wavelet packet and FHN model, signal is denoised by the piecewise threshold wavelet packet firstly, then the obtained signal is processed by FHN model stochastic resonance to realize the waveform reconstruction of the UWB-IR signal. Simulation result shows that the denoising performance of the piecewise threshold wavelet packet is better than the traditional single threshold wavelet packet, the UWB-IR signal can be detected from the strong noise effectively by the wavelet packet and FHN model joint detection method. Compared with the detection method that using FHN model only, the signal characteristics can be judged better from the reconstructed signal and the UWB-IR signal can be detected from a lower SNR environment by using the joint detection method, so the proposed method improve the performance of FHN model, it is suitable to detect the UWB-IR signal under strong noise.

\section{Wavelet packet}

Wavelet transform has a good time-frequency characteristics, however, wavelet transform is used to decompose the low frequency part of the signal only, which is easy to ignore the useful information in the high frequency, lead to denoise excessively and useful information can't be extracted effectively. Wavelet packet can subdivide both the low frequency and the middle, high frequency component of the signal, useful information can be extracted relatively and efficiently.

\subsection{Basic theory of wavelet packet}

The formula of wavelet packet decomposition [14] is as follows:

$$
\left\{\begin{array}{c}
d_{j}^{2 n}=\sum_{k \in Z} h_{k-2 l} d_{j+1, k}^{n} \\
d_{j}^{2 n+1}=\sum_{k \in Z} g_{k-2 l} d_{j+1, k}^{n}
\end{array}\right.
$$

Where: $d_{j}$ is wavelet packet coefficients.

Wavelet packet reconstruction formula is as follows:

$$
d_{j+1}^{n}=\sum_{k \in Z}\left(h_{l-2 k} d_{j k}^{2 n}+g_{l-2 k} d_{j k}^{2 n+1}\right)
$$


Where: $h$ and $g$ is filter coefficients, $j$ and $n$ is wavelet packet node number, $k$ and $l$ is decomposition layer.

\subsection{Wavelet packet decomposition}

The essence of wavelet packet decomposition is signal processed by a set of high/low pass conjugate filters, the frequency will flip when signal pass through the high pass filter. Therefore, the frequency dislocation is occurred in the process of signal decomposition, that is, the natural node order of is inconsistent with the frequency order[15]. Take the 3 layer wavelet packet decomposition as an example, the natural node order and the frequency order are shown in Fig.1.

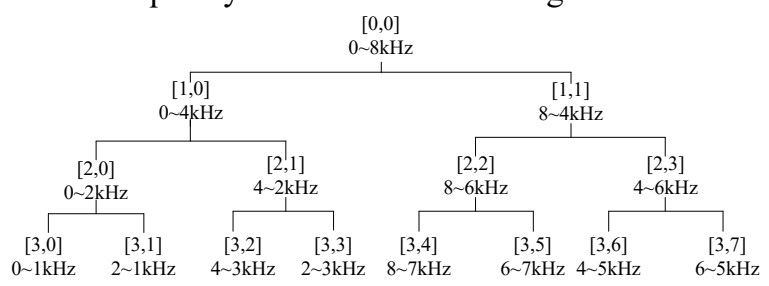

Fig. 1 Three layer wavelet packet decomposition

\subsection{Traditional wavelet packet threshold criterion}

Threshold criterion selection is a key in the process of wavelet packet denoising, which is related to the performance of signal denoising directly. The traditional four threshold criterion is sqtwolog, heursure, minimaxi and rigrsure[16]. Sqtwolog and the heursure process all the wavelet packet coefficients, so this two kinds of threshold criteria have strong denoising ability, but excessive denoising will be occurred easily; minimaxi and rigrsure process partial wavelet packet coefficients, so the denoising process is mild, it is suitable to process signal in low frequency. Four threshold criteria are shown as follows respectively:

(1) Sqtwolog use a fixed form expression, the formula is as follows:

$$
T_{1}=\sigma \sqrt{2 \ln N}
$$

Where: $\sigma$ is the standard deviation of noise, $N$ is the length of signal.

(2) Rigrsure is a threshold criterion based on Stein unbiased likelihood estimation, the formula is as follows:

$$
T_{2}=\sigma \sqrt{Q_{a}}
$$

Where: $\sigma$ is the standard deviation of noise, $Q_{a}$ is the square of decomposition coefficient based on unbiased likelihood estimation.

(3) Heursure threshold criterion integrate two threshold criterion that is mentioned above, it is an unbiased risk threshold, the formula is as follows:

$$
T_{3}= \begin{cases}T_{1} & \alpha>\beta \\ T_{2} & \alpha<\beta\end{cases}
$$

Where: $\alpha=(W-N) / N ; \beta=\sqrt[3 / 2]{\left(\log _{2} N\right) N} ; W$ is the sum of $N$ wavelet packet decomposition coefficient squares.

(4) Minimaxi use a fixed form expression too, it find the minimum value of the maximum mean square error in the worst case, which is called "pessimistic" in statistics ,the formula is as follows:

$$
T_{4}= \begin{cases}\sigma\left(0.3936+0.182 \log _{2} N\right) & N>32 \\ 0 & N<32\end{cases}
$$

Where: $\sigma$ is the standard deviation of noise, $N$ is the length of signal.

\subsection{Piecewise wavelet packet threshold criterion}

Four threshold criteria that was described in the previous have their own suitable frequency ranges, wavelet packet denoising effect will not be obvious if the frequency beyond the suitable range. In practical application, however, noise often distributed in a high frequency band relatively than the useful signal, if single threshold criterion is used to process the wavelet packet coefficients will lead to excessive denoising or incomplete denoising. In view of this, a new piecewise threshold criterion is proposed, that is, the sub bands that were decomposed by wavelet packet are rearranged from low to high frequency firstly, then the sub bands are divided into three bands: low frequency, medium frequency and high frequency, the wavelet packet coefficients of different frequency bands are processed by suitable threshold criterion, finally, output the reconstructed signal. A $n$ layer wavelet packet decomposition as an example, assuming that the lowfrequency coefficient is $l$, and the high-frequence coefficient is $h$, frequency bands division criteria is as follows: 


$$
\left\{\begin{array}{lr}
2^{n} \times l & \text { low frequency } \\
2^{n} \times(1-l-h) & \text { medium frequency } \\
2^{n} \times h & \text { high frequency }
\end{array}\right.
$$

According to the frequency band division criterion, the selection of the threshold criterion is shown in Table 1.

Table 1. Threshold criterion selection

\begin{tabular}{ccc}
\hline $\begin{array}{c}\text { Low } \\
\text { Frequency }\end{array}$ & $\begin{array}{c}\text { Medium } \\
\text { Frequency }\end{array}$ & $\begin{array}{c}\text { High } \\
\text { Frequency }\end{array}$ \\
\hline rigrsure & rigrsure & sqtwolog \\
minimaxi & minimaxi & heursure \\
\hline
\end{tabular}

\section{UWB-IR Detection Algorithm Based on Wavelet Packet and FHN Model}

FHN neuron model is simplified on the basic of the H-H model, the neuron can amplify the weak signal by the stochastic resonance in the process of information coding and transmission under noise enviroment.

\subsection{FHN neuron model}

FHN neuron model stochastic resonance is represented by the equations as follows[17]:

$$
\left\{\begin{array}{l}
\varepsilon \frac{\mathrm{d} x}{\mathrm{~d} t}=x(x-\mathrm{a})(1-x)-y+\mathrm{A}+s(t)+n(t) \\
\frac{\mathrm{d} y}{\mathrm{~d} t}=x-y-\mathrm{b}
\end{array}\right.
$$

Where: $x$ is the fast variable of neuron membrane voltage, $y$ is recovery variable, $\varepsilon$ is time constant, $a$ and $b$ is the constant of the equation group, $b$ is taken 0.01 generally; $\mathrm{A}$ is the critical threshold constant, take $0.11 \mathrm{mV}$ generally; $s(t)$ is UWB-IR signal, $n(t)$ is gauss white noise with zero mean. The four order Runge-Kuta algorithm is usually adopted to solve this differential equations. The research result of shows that $a$ and $\varepsilon$ will affect the performance of FHN neuron model to detect UWB signal, their values should be adjusted dynamically according to the different detection signal.

\subsection{Evaluation criteria of FHN model}

Input and output cross-correlation function can be adopted as the evaluation criteria of FHN model, it has been proved that calculate the maximum value of the input and output cross correlation function can achieve the best detection, the formula is as follows:

$$
C_{1}=\frac{\langle[\eta(t)-\overline{\eta(t)}] \cdot[R(t)-\overline{R(t)}]\rangle}{\sqrt{\left\langle[\eta(t)-\overline{\eta(t)}]^{2} \cdot[R(t)-\overline{R(t)}]^{2}\right\rangle}}
$$

Where: $\eta(t)$ is the input signal with noise, $R(t)$ is the output of FHN model stochastic resonance.

\subsection{Parameter optimization of FHN model system}

The value of $a$ and $\varepsilon$ will affect the performance of FHN model to detect UWB signal, their values should be adjusted dynamically according to the different detection signal, for that reason, Quantum Particle Swarm Optimization(QPSO)[18] is introduced into FHN model to optimize the parameter of FHN model dynamically.

The main steps of the QPSO are as follows:

Step 1 Initialization of QPSO parameter. Initialize the maximum number of iterations $T_{\max }$, population quantity $M$, dimension and the scope of each dimension. Initialize the particle position randomly within the limits, as shown in formula (10):

$$
x_{i}=\left(x_{i 1}, x_{i 2}, \cdots x_{i D_{\mathrm{dim}}}\right) \quad i=1,2, \cdots, M
$$

Step 2 Initialize the optimal fitness value. Calculated the fitness value of each particle according to formula (9), then take the fitness values of the first generation particles as the local optimal fitness values and record as $p_{\text {best }}(i), i=1,2, \cdots, M$, select the maximum value as the global optimal fitness value and record $g_{\text {best }}$.

Step 3 Update the optimal fitness. Update particle position according to formula (11), then calculate the fitness value of each particle position after updated, update the local optimal fitness value or the global optimal fitness value if the new $p_{\text {best }}(i)$ or $g_{\text {best }}$ is better than the previous generation, Particle update formula is as follows:

$$
x(t+1)=P \pm \beta\left|m_{\text {best }}-x(t)\right| \ln (1 / u)
$$

Where $P$ is the global optimal position for the iteration to $t ; u$ is the random number on $[0,1], \beta$ is negative when $u \leq 0.5$, otherwise $\beta$ is positive, The expression of $\beta$ is shown in the formula (12): 


$$
\beta=0.5+\frac{(1-0.5)\left(T_{\max }-t\right)}{T_{\max }}
$$

Where $\boldsymbol{m}_{\text {best }}$ is the average best position of all particles in the particle swarm when iteration number is $t$, the formula is as follows:

$$
m_{\text {best }}=\sum_{i=1}^{M} P_{i} / M=\left(\sum_{i=1}^{M} P_{i 1} / M, \sum_{i=1}^{M} P_{i 2} / M, \cdots \sum_{i=1}^{M} P_{i D_{-}} / M\right)
$$

Step 4 Output the optimal result. Obtain the optimal parameter values according to the global optimal fitness, then plug into the optimal parameter into formula (8) to obtain the optimal output results.

\subsection{UWB-IR detection algorithm based on wavelet packet and FHN model}

In order to overcome the SNR threshold of FHN neural network model for detecting UWB-IR signal and improve the performance of it, a UWB-IR detection method based on wavelet packet and FHN neuron model is proposed. Firstly, the piecewise wavelet packet is used to preprocess the noisy UWB-IR signal; then the signal that was denoised by piecewise wavelet packet is used as the input of the FHN neuron model stochastic resonance system, and QPSO is used to optimize the parameters of FHN model; Finally obtain the reconstructed UWB-IR signal waveform. The algorithm flow chart is shown in Fig.2.

\section{Simulation experiment}

\subsection{Ultra-wide band impulse signal model}

The expression of the UWB-IR signal is chosen as the simulation test:

$$
\begin{aligned}
& s(t)=\mathrm{A} \sum_{k}\left(\left[1-0.4 \pi\left(\frac{t-\mathrm{t}_{0}-k \mathrm{~T}}{\mathrm{t}_{n}}\right)^{2}\right]\right. \\
&\left.\exp \left[-0.2 \pi\left(\frac{t-\mathrm{t}_{0}-k \mathrm{~T}}{\mathrm{t}_{n}}\right)^{2}\right]\right) \\
& k \in\{0,1,3,5,6,8\}(14)
\end{aligned}
$$

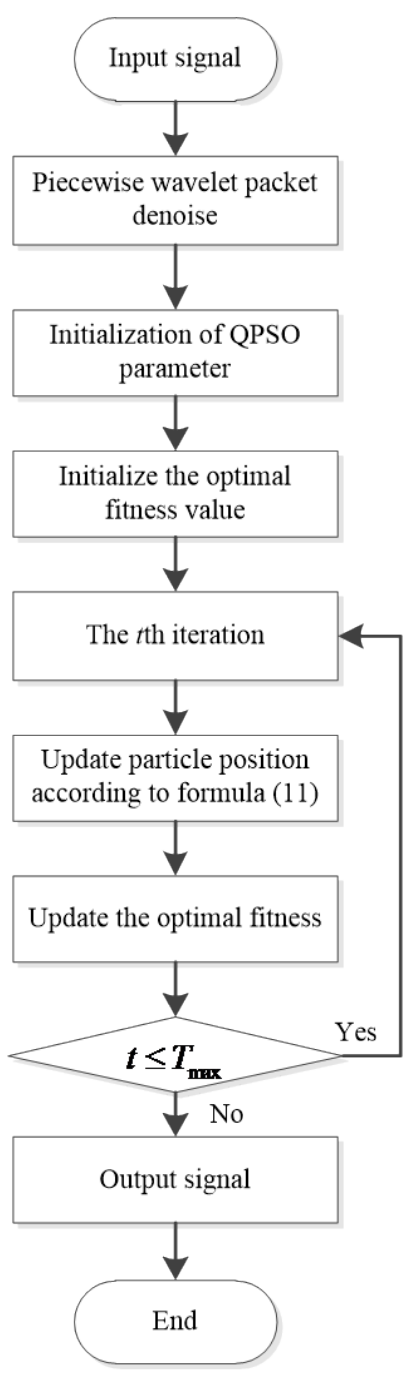

Fig. 2 Algorithm flow chart

In formula (20): $t=1: 1: 1000 \mathrm{~ns}$, parameters of Gauss's two order derivative impulse function $t_{n}=3.112 \mathrm{~ns}$, $\mathrm{t}_{0}=8.51 \mathrm{~ns}, \mathrm{~T}=9.8 \mathrm{~ns}$, A is signal amplitude. Formula (14) is a non-stationary nonlinear signal, which is composed of two-order derivatives of Gauss's function, When $\mathrm{A}=1.141 \mathrm{~V}$, the signal waveform is shown in Fig.3.

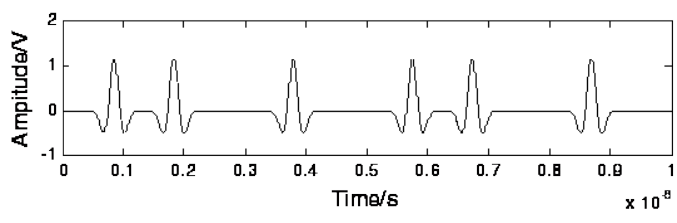

Fig. 3 UWB-IR signal 


\subsection{Piecewise threshold wavelet packet}

In order to verify the effectiveness of the piecewise wavelet threshold denoising algorithm that is proposed, UWB-IR signal is processed by single threshold wavelet packet and piecewise threshold wavelet packet respectively under different SNR condition, Root mean square error(RMSE) is adopted as the method for measuring performance of algorithm, RMSE is expressed as:

$$
R M S E=\sqrt{\frac{1}{n} \sum_{i=1}^{n}\left[s(i)-s^{\prime}(i)\right]^{2}}
$$

Where: $s(i)$ is the original UWB-IR signal, $s^{\prime}(i)$ is the signal after wavelet packet noise denoising, $n$ is the length of signal. In the simulation, $\mathrm{db} 4$ wavelet is selected to discompose signal in five layers, low frequency ratio coefficient $\alpha$ is $1 / 4$, high frequency ratio coefficient $\beta$ is $1 / 4$, entropy criterion is SURE entropy. Sqtwolog, minimaxi and rigrsure is adopted as the threshold criterion of high frequency, middle frequency and low frequency respectively, the simulation experiment is executed 50 times to each threshold criterion and each SNR, and take the average value of RMSE, the experimental results shown in Fig.4.

It can be seen from Fig. 4 that the RMSE of piecewise threshold wavelet is smaller than other traditional single threshold wavelet packet, therefore, the piecewise threshold wavelet can denoise the excess noise effectively and retain the useful information as much as possible.

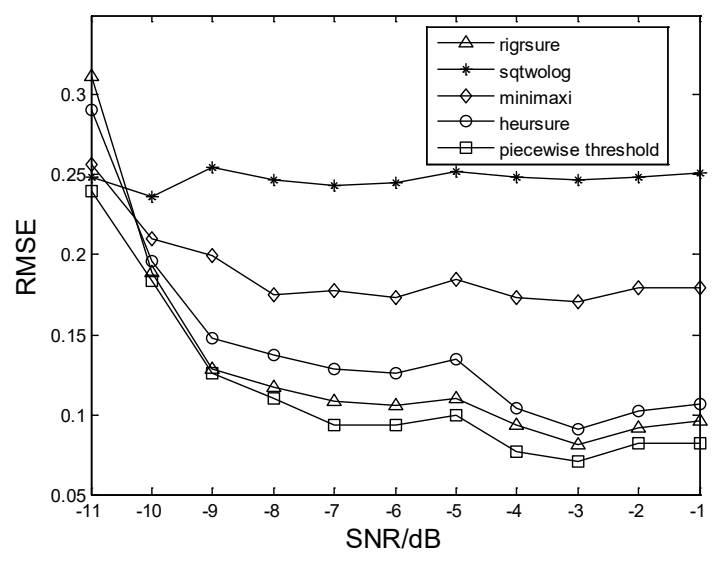

Fig. 4 Comparison of different threshold criteria

\subsection{Joint detection method based on wavelet packet and FHN model}

Joint the wavelet packet and FHN model to detect the UWB-IR signal, which makes the UWB-IR signal can be detected under a lower SNR condition. When SNR is $-21.5 \mathrm{~dB}$, the detection result of wavelet packet and FHN model is showed in Fig.5.
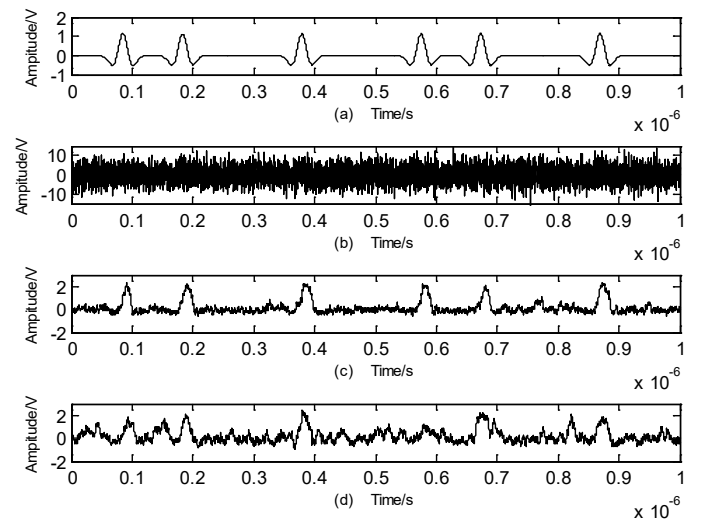

Fig. 5 Comparison of detection result

Fig.5(a) is the original UWB-IR signal, Fig. 5(b) is the UWB-IR signal when SNR is $-21.5 \mathrm{~dB}$, Fig.5(c) is the signal reconstructed waveform detected by wavelet packet and FHN model, Fig5(d) is the reconstructed signal waveform that was only detected by FHN model.

The simulation result shows that UWB-IR signal can be detected by wavelet packet and FHN model joint method effectively when SNR is $-21.5 \mathrm{~dB}$, the output signal is able to determine the pulse position information of the UWB-IR signal accurately. In addition, partial noise energy is transmitted to the UWB-IR signal after the stochastic resonance of the FHN neuron model due to the characteristics of nonlinear stochastic resonance, which further improve the detection performance. According to the literature[14], the SNR threshold that UWB-IR detected by FHN neuron model is $-20 \mathrm{~dB}$, when SNR is $-21.5 \mathrm{~dB}$, the fitting degree between the detected signal and the original signal is low, and the signal waveform cannot be recovered accurately, as shown in Fig.6(d). Therefore, compared with the method that using the FHN model to detect UWB-IR signal only, UWB-IR can be detected from lower SNR by wavelet packet and FHN model joint detection effectively, feature information of UWB-IR signal can be extracted better. 
In order to verify the superiority of the wavelet packet and FHN model joint method that proposed to detect UWB-IR signal, the proposed method and FHN model are used to detect the UWB-IR signal at different SNR, the simulation experiment is executed 100 times at each SNR, then record the average value of RMSE and input and output cross correlation function. The simulation results are shown in Fig.6and Fig.7.

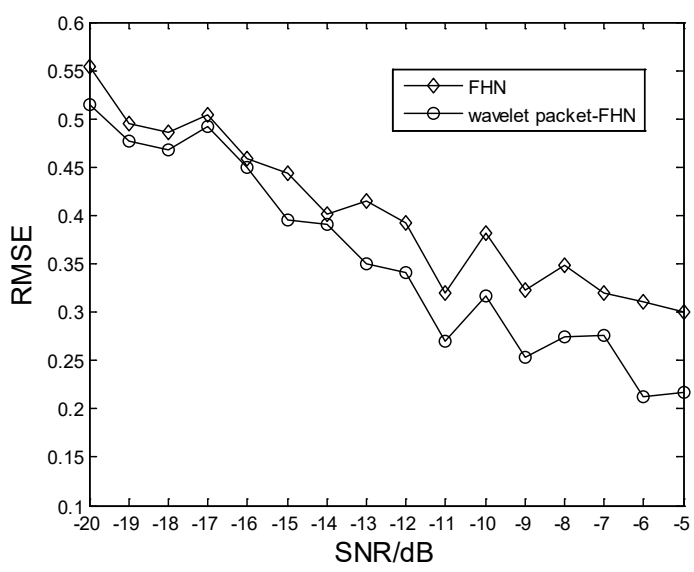

Fig. 6 Comparison of RMSE in two methods

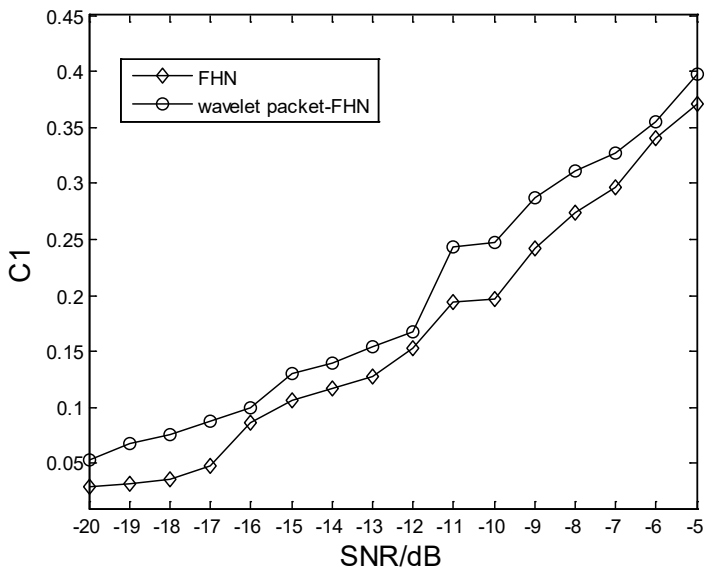

Fig. 7 Comparison of Input-output cross correlation in two methods

From Fig. 6 and Fig.7 can be seen that under the same condition of SNR, the RMSE that detect UWB-IR signal by wavelet packet and FHN model joint method is significantly less than the RMSE that obtained the FHN model detection method only, input output cross correlation function value of the proposed method is greater than the FHN model method, so the proposed method improve the performance of the FHN model to detect the UWB-IR signal effectively, it is a suitable method to detect the UWB-IR signal.

\section{Conclusion}

In order to improve the ability of FHN neural model to detect UWB-IR signal under strong noise environment, a wavelet packet and FHN model joint method is proposed, that is the wavelet packet is used denoise the signal firstly, then the FHN model is used to reconstruct the signal. Furthermore, a piecewise threshold wavelet packet is proposed to improve the performance of traditional wavelet packet, which joint with the model, the ability of the FHN model to detect the UWB-IR signal is extended to a lower SNR. Simulation experiment shows that compared with traditional single threshold wavelet packet, the piecewise threshold wavelet packet can retaining useful information in the signal as much as possible in the process of denoising. In the simulation experiment, the signal that is denoised by piecewise threshold wavelet packet and the signal that didn't processed by wavelet packet is reconstructed by FHN model respectively, the simulation result shows that the piecewise threshold wavelet packet combined with FHN model can reduce the SNR in UWB-IR signal detection, improve the detection performance of the FHN model, the SNR can reach to $-21.5 \mathrm{~dB}$. In addition, we also use the values of RMSE and the input and output cross correlation to illustrate the superiority of the wavelet packet and FHN model joint method. Hence, the UWB-IR signal can be detected by the proposed wavelet packet and FHN model joint can detect under a lower SNR, which is a suitable method to detect UWB-IR signal under strong noise.

\section{Acknowledgment}

This research is supported by the National Natural Science Foundation of China (Grant No.61471386).

\section{References}

1. F Q Wang, X F Zhang, F Wang. Root-MUSIC-based joint TOA and DOA estimation in IR-UWB. Journal on Communications, 2014(2):137-145. 
2. D Li, F B Jang. A Novel Multi-Strategy Routing for UWB Ad Hoc Networks. Advances in Intelligent \& Soft Computing, 124 (2011)25-30.

3. Lim J M, Park J W, Lee K J, et al. Comparison of localization methods using CSS-UWB WPAN for mobile robots, International Conference on Ubiquitous Robots and Ambient Intelligence. (2012), pp. 347-349.

4. Y Li. Research on ultra-wideband short range inpulse imagine radar system. University of Electronic Science and Technology of China, (2014).

5. X Wang, L Lin, $\mathrm{H}$ Tang, et al. Low power $3.1-10.6 \mathrm{GHz}$ IR-UWB transmitter for Gbps wireless communications. Science China Information Sciences, 54(5)(2011)10941102.

6. H W Deng, Y J Zhao, F Fu, et al. Compact and High Isolation Microstrip Diplexer for GPS and UWB Application. Electeonics Letters, 49(10)(2013)659-666.

7. X S Li, T Q Zhang, L F Yang, et al. The Period Estimation of PN Sequence Using Reprocessing Spectrum of DSUWB signal, International Conference on Communication Software and Networks. IEEE, (2009), pp. 270-274.

8. X W Liu, L Jiang, H Xu. Study on end effects of EMD with HHT for UWB signal detection[C]// 2015 International Conference on Estimation, Detection and Information Fusion (ICEDIF). 2015.

9. Q An, Z Li, F L Liang. et al. Wavelet Based human target detection in complex ruins using a low center frequency UWB radar, 2016 Progress in Electromagnetic Research Symposium . (2016).

10. Z Y Xi, Y J Yan, Y Zhang. et al. Stochastic Resonance and its Application in Detecting Weak Signal, 2010 3rd International Congress on Image and Signal Processing, IEEE, (2010), pp. 4148-4151.
11. L L Wu, G H Hui, A Y Lin. et al. Study on early moldy grain identification based onaperiodic stochastic resonance. Chinese Journal of Scientific Instrument, 32(4)(2011)742-747.

12. X W Liu, L Jiang. Utlra-wideband signal detection based on FHN model method. Journal of Air Force Engineering University (Natural Science Edition), 2015(4)(2015)58-61.

13. Y X Sun, Z H Xiong. An Optimal Weighted Wavelet Packet Entropy Method With Application to Real-Time Chatter Detection. IEEE/ASME Transactions on Mechatronics, , 21(4)(2016)2004-2014.

14. H Chen, ASSALA P, Y Cai, et al. Intelligent Transient Overvoltage Location in Distribution Systems Using Wavelet Packet Decomposition and General Regression Neural Networks. IEEE Transactions on Industrial Informatics, 2016(2016)1-1.

15. FATHI A, NAGHSH-NILCHI A R. Efficient image denoising method based on a new adaptive wavelet packet thresholding function. IEEE Transactions on Image Processing A Publication of the IEEE Signal Processing Society, 21(9) (2012)3981-90.

16. J D Mao, D X Hua, Y F Wang. Noise Reduction in Lidar Signal Based on Wavelet Packet Analysis. Chinese Journal of Lasers, 2011(2)(2011)220-227.

17. K Chen, Y L Fan, L S Geng. The Research on the Stotistic Resonance Based of Feedback FitzHugh-Nagumo Neural Network, World Congress on Intelligent Control and Automation. IEEE, (2010), pp. 6729-6734.

18. H G Li, X L Zhang, H M Zhang. An improved actuator node deployment algorithm of CPSs network based on QPSO, International Conference on Information Science \& Technology. IEEE, (2016). 